\title{
A hint of Poincaré dodecahedral topology in the WMAP first year sky map
}

\author{
B. F. Roukema ${ }^{1}$, B. Lew ${ }^{1}$, M. Cechowska ${ }^{1}$, A. Marecki ${ }^{1}$, and S. Bajtlik ${ }^{2}$ \\ 1 Toruń Centre for Astronomy, N. Copernicus University, ul. Gagarina 11, 87-100 Toruń, Poland \\ e-mail: boud@astro.uni.torun.pl \\ 2 Nicolas Copernicus Astronomy Centre, ul. Bartycka 18, 00-716 Warsaw, Poland
}

Received 25 February 2004 / Accepted 26 May 2004

\begin{abstract}
It has recently been suggested by Luminet et al. (2003) that the WMAP data are better matched by a geometry in which the topology is that of a Poincaré dodecahedral model and the curvature is "slightly" spherical, rather than by an (effectively) infinite flat model. A general back-to-back matched circles analysis by Cornish et al. (2004) for angular radii in the range $25-90^{\circ}$, using a correlation statistic for signal detection, failed to support this. In this paper, a matched circles analysis specifically designed to detect dodecahedral patterns of matched circles is performed over angular radii in the range $1-40^{\circ}$ on the oneyear WMAP data. Signal detection is attempted via a correlation statistic and an rms difference statistic. Extreme value distributions of these statistics are calculated for one orientation of the $36^{\circ}$ "screw motion" (Clifford translation) when matching circles, for the opposite screw motion, and for a zero (unphysical) rotation. The most correlated circles appear for circle radii of $\alpha=11 \pm$ $1^{\circ}$, for the left-handed screw motion, but not for the right-handed one, nor for the zero rotation. The favoured six dodecahedral face centres in galactic coordinates are $\left(l^{\mathrm{II}}, b^{\mathrm{II}}\right) \approx\left(252^{\circ},+65^{\circ}\right),\left(51^{\circ},+51^{\circ}\right),\left(144^{\circ},+38^{\circ}\right),\left(207^{\circ},+10^{\circ}\right),\left(271^{\circ},+3^{\circ}\right),\left(332^{\circ},+25^{\circ}\right)$ and their opposites. The six pairs of circles independently each favour a circle angular radius of $11 \pm 1^{\circ}$. The temperature fluctuations along the matched circles are plotted and are clearly highly correlated. Whether or not these six circle pairs centred on dodecahedral faces match via a $36^{\circ}$ rotation only due to unexpected statistical properties of the WMAP ILC map, or whether they match due to global geometry, it is clear that the WMAP ILC map has some unusual statistical properties which mimic a potentially interesting cosmological signal.
\end{abstract}

Key words. cosmology: observations - cosmology: cosmic microwave background - reference systems

\section{Introduction}

In the past twelve months, several authors have analysed the possibility that the primordial temperature fluctuations in the cosmic microwave background, as measured in the first-year data of WMAP (Wilkinson Microwave Anisotropy Probe) satellite (Spergel et al. 2003, and accompanying papers), could be better matched by a perturbed Friedmann-Lemaître-Robertson-Walker (FLRW) model in which the global geometry is multiply connected rather than simply connected.

The possibility of the Universe being multiply connected is first known to have been suggested by Schwarzschild (1900, 1998). For recent reviews on cosmic topology, see Lachièze-Rey \& Luminet (1995), Luminet (1998), Starkman (1998) and Luminet \& Roukema (1999). For workshop proceedings on the subject, see Starkman (1998) and following articles, and Blanlœeil \& Roukema (2000). Detection strategies include both two-dimensional methods (based on temperature fluctuations in the surface of last scattering) and three-dimensional methods (based on distributions of gravitationally collapsed objects distributed in three-dimensional comoving space). For a list and discussion of both twodimensional and three-dimensional methods, see Table 2 of Luminet \& Roukema (1999) and the accompanying discussion.

The WMAP data has motivated many two-dimensional analyses.

Some of the authors mentioning either multiply connected models consistent with the WMAP data, or indirect hints of multiple connectedness, include Spergel et al. (2003); Tegmark et al. (2003); Chiang et al. (2003); Copi et al. (2003), - finding low values of low $l$ multipoles or applying a multipole vector analysis.

However, other authors (e.g., Feng \& Zhang 2003; Cline et al. 2003; Contaldi et al. 2003; Efstathiou 2003a) have suggested various non-topological explanations for the low multipole WMAP $C_{1}$ spectrum, such as double inflation or other phenomena from early universe physics, or positive curvature.

Yet others (Naselsky et al. 2003, 2004) point out that at least the "internal linear combination" (ILC) map of the WMAP data contains non-Poissonian signal, due to foreground residues on large scales. On smaller scales, Giommi \& Colafrancesco (2004) find that somewhere between $20-100 \%$ of the signal at 
spherical harmonic $l$ values in the range $500<l<800$ may be due to blazars.

Attempts to exclude classes of global geometry models using the WMAP data include the calculations of de OliveiraCosta et al. (2004) and of Cornish et al. (2004). The latter performed a general back-to-back matched circles analysis for angular radii in the range $25-90^{\circ}$, using a correlation statistic for signal detection. They failed to find matched circles for a wide class of models, including the torus models, up to a scale of $16.8 h^{-1} \mathrm{Gpc}$, which Cornish et al. (2004) state as $24 \mathrm{Gpc}$, since they adopt $H_{0}=70 \mathrm{~km} \mathrm{~s}^{-1} \mathrm{Mpc}^{-11}$.

Disagreement also exists on whether or not the low quadrupole is really significant in rejecting the infinite flat "concordance" model (Efstathiou 2003b, 2004).

Possibly one of the strongest claims in favour of a possible detection is that by Luminet et al. (2003), who point out that given standard assumptions on the statistics of the fluctuations, the Poincaré dodecahedral model implies a quadrupole and an octupole very close to those calculated from the WMAP data, for the same value of the total density parameter, $\Omega_{\mathrm{tot}} \approx 1.013 \pm$ 0.02 .

In principle, this is excluded by the Cornish et al. (2004) analysis.

The Poincaré dodecahedral model requires positive (spherical) curvature. Luminet et al. (2003) favour a total density parameter of $\Omega_{\mathrm{tot}} \approx 1.013 \pm 0.002$ based on the spherical harmonic statistical analyses of the WMAP data, with nonrelativistic matter density parameter, $\Omega_{\mathrm{m}}=0.28$ and cosmological constant $\Omega_{\Lambda}=\Omega_{\mathrm{tot}}-\Omega_{\mathrm{m}}$. This implies that points on the surface of last scattering which are multiple topological images of single physical points in space-time should correspond to matched circles (Cornish et al. 1996, 1998) which subtend angular radii at the observer of "about $35^{\circ}$ " (Luminet et al. 2003).

Since the angular relations between face centres are identical for Euclidean and spherical dodecahedra, Euclidean calculations of the relative positions of circles are sufficient for testing the Poincaré dodecahedral hypothesis. For example, the Euclidean half-angle of about $31.7^{\circ}$ is valid for angular separations of adjacent face centres in the spherical dodecahedron. Since the adjacent circles expected by Luminet et al. (2003) have larger angular radii (subtended at the observer), this implies that they intersect with the face edges.

However, as is shown in Sect. 3.2, this angular radius is extremely sensitive to the value of $\Omega_{\text {tot }}$. For example, keeping $\Omega_{\mathrm{m}}=0.28$ fixed, it is sufficient to decrease $\Omega_{\mathrm{tot}}$ to $\Omega_{\mathrm{tot}}=1.009$ to bring the angular radius to nearly zero.

This implies that a Poincaré dodecahedral signal may have been missed by Cornish et al. (2004) because they did not explore the part of parameter space for small matched circles.

In this paper, the missing part of parameter space is investigated. A matched circles analysis specifically designed to detect dodecahedral patterns of matched circles is performed over angular radii in the range $1-40^{\circ}$ on Internal Linear Combination map (ILC) of the one-year WMAP data. The WMAP data are briefly discussed in Sect. 2. While the ILC

\footnotetext{
1 The Hubble constant is parametrised as $h \equiv H_{0} / 100 \mathrm{~km} \mathrm{~s}^{-1} \mathrm{Mpc}^{-1}$.
}

is unlikely to be ideal for the studies of $C_{1}$ statistics, and some authors (cited above) claim correlations with foregrounds, it is hard to see how any signal mimicking matched circles oriented in a dodecahedral pattern could be imposed, either by the construction method of the ILC or by foregrounds.

Signal detection is attempted via a correlation statistic and an rms difference statistic, similarly to Roukema (2000a,b). Extreme value distributions of these statistics between a righthanded rotation when matching circles, a left-handed rotation, and a zero rotation. A genuine signal should appear for either the right-handed or left-handed rotation, but not both, and should not appear for the zero rotation.

The relation between circle angular radius $(\alpha)$ and local cosmological parameters $\left(\Omega_{\mathrm{m}}, \Omega_{\mathrm{tot}}\right)$ and the statistics used are presented in Sect. 3.

The tentative detection of dodecahedrally distributed matched circles with $\alpha \approx 10^{\circ}$ and analysis of their statistical significance are presented in Sect. 4. Further discussions and conclusions are made in Sect. 5.

For reviews on cosmological topology, see Lachièze-Rey \& Luminet (1995), Luminet (1998), Starkman (1998) and Luminet \& Roukema (1999). For workshop proceedings on the subject, see Starkman (1998) and the following articles, and Blanlœil \& Roukema (2000). For a list and discussion of both two-dimensional and three-dimensional methods, see Table 2 of Luminet \& Roukema (1999) and the accompanying discussion. The reader should be reminded that while microwave background data is still the most popular for topology analyses, considerable work in three-dimensional methods has been carried out, including, e.g., Lehoucq et al. (1996); Roukema (1996); Fagundes \& Gausmann (1998b); Roukema \& Edge (1997); Roukema \& Blanlœil (1998); Gomero et al. (2002); Lehoucq et al. (1999); Fagundes \& Gausmann (1998a); Uzan et al. (1999); Fagundes \& Gausmann (1999); Gomero et al. (2000, 2001).

For background on spherical multiply connected spaces, apart from the recent analysis by Luminet et al. (2003), see Gausmann et al. (2001); Lehoucq et al. (2002); Riazuelo et al. (2003) for extremely thorough, in-depth mathematical background directly related to the cosmological context. For a general background on geometry and topology, see e.g. Weeks (2001).

Comoving coordinates are used when discussing distances (i.e. "proper distances", Weinberg 1972, equivalent to "conformal time" if $c=1$ ).

\section{Observations}

See Spergel et al. (2003) and accompanying papers for an introduction to the WMAP data.

While some authors make their own combinations of the WMAP maps in individual frequencies, it is hard to see how any signal mimicking matched circles oriented in a dodecahedral pattern could be imposed, by the method according to which the Internal Linear Combination (ILC) map is constructed. So this is the data set used here. 
However, in order to obtain conservative results, all points within $2^{\circ}$ of the galactic plane or $20^{\circ}$ of the galactic centre are removed from the analysis.

Moreover, a smoothing of $1^{\circ} \mathrm{HWHM}$ is applied for the main calculation, since if a signal is present, it should be present both on super-degree scales and on sub-degree scales, but should be easier to detect on the larger scales, because the search calculations can be made much faster, because the naïve Sachs-Wolfe dominates over the Doppler effect on larger scales, and because there is likely to be less influence from subtle effects which need to be taken into account as correction factors, e.g. the special relativistic correction for the observer's motion with respect to the microwave background, Barrow \& Levin (2001); Uzan et al. (2002).

\section{Method}

\subsection{The identified circles principle}

The identified circles principle was first published by Cornish et al. $(1996,1998)$. This defines the set of multiply topologically imaged points for a given manifold.

This set can be generated by considering copies of the observer in the covering space placed at distances less than the horizon diameter from the observer. The intersection of the two surfaces of last scattering (spheres) of the observer and a copy of the observer is a circle. Since the copy of the observer is physically identical to the observer, what appears to be two observers looking at one circle is equivalent to one observer looking at two circles.

Hence, each pair of virtual copies of the observer in the covering space (separated by less than the diameter of the surface of last scattering) implies a pair of circles on the surface of last scattering which correspond to identical points in space-time.

\subsection{Relating circle angular radius $\alpha$ to $\Omega_{m}, \Omega_{\text {tot }}$}

As mentioned above, Euclidean geometry is sufficient for calculating the positions of matched circles for the Poincaré dodecahedral hypothesis of Luminet et al. (2003), even though the model is for positive curvature. Errors would occur only if, for example, the angular radius of a circle, $\alpha$, is converted into a comoving distance in megaparsecs, or if the ratio of the inverse cosine of the ratio of the in-radius, $r_{-}$, to the distance to the surface of last scattering, $r_{\mathrm{SLS}}$, is used to infer the angular radius of a matched circle, i.e.

$\alpha \approx \cos ^{-1}\left(\frac{r_{-}}{r_{\mathrm{SLS}}}\right)$.

Since the values of $\Omega_{\mathrm{tot}}$ of interest are very close to unity, either of these calculations should only lead to small errors.

In fact, a much bigger uncertainty is due to the sensitivity of $\alpha$ to the values of $\Omega_{\mathrm{tot}}$ and $\Omega_{\mathrm{m}}$.

The curvature radius can be defined in a strict sense, without taking an absolute value of the curvature, as

$R_{\mathrm{C}}=\frac{c}{H_{0}} \frac{1}{\sqrt{\Omega_{\mathrm{tot}}-1}}$.

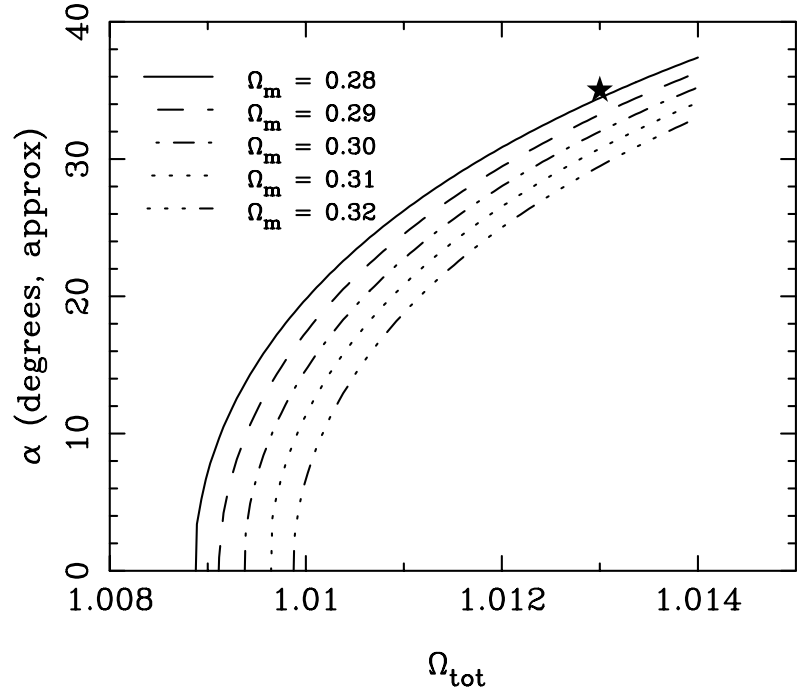

Fig. 1. Dependence of the angular size $\alpha$ of matched circle radii on the total density parameter $\Omega_{\text {tot }}$ and the non-relativistic matter density parameter $\Omega_{\mathrm{m}}$ for the Poincaré dodecahedral hypothesis. Clearly only slight changes in $\Omega_{\text {tot }}$ are needed for the circle radius to change significantly. The values $\Omega_{\mathrm{tot}}=1.013, \alpha=35^{\circ}$ expected by Luminet et al. (2003) are shown by a star. The word "approx" is used in the label to remind the reader that this is not an exact calculation, since Euclidean geometry is assumed.

This has the advantage of showing that the transition from "slightly" hyperbolic space to flat space to spherical space, i.e. from $\Omega_{\text {tot }}<1$ to $\Omega_{\text {tot }}=1$ to $\Omega_{\text {tot }}>1$ starts from imaginary values of the curvature radius, increases to larger and larger imaginary curvature radii without bounds, goes through a singularity, and decreases from unbounded large, real values to smaller and smaller real values as $\Omega_{\text {tot }}$ increases through positive values.

It is therefore unsurprising that other physical quantities related to the curvature radius may show rapid changes near the flatness limit.

To illustrate the relation between $\alpha, \Omega_{\text {tot }}$ and $\Omega_{\mathrm{m}}$, the value of $r_{-}$, the in-radius of the fundamental domain of the Poincaré dodecahedron, expressed as

$r_{-}=0.31 R_{\mathrm{C}}$

is used, along with Eq. (1) and the comoving distance to the surface of last scattering

$r_{\mathrm{SLS}}=r\left(\Omega_{\mathrm{m}}, \Omega_{\mathrm{tot}}, H_{0}, z=1100\right)$.

Figure 1 shows how a few tenths of a percent drop in $\Omega_{\text {tot }}$ are sufficient to increase the curvature radius so that the in-radius of the fundamental dodecahedron becomes as large as the surface of last scattering, reducing the circle size from $\approx 35^{\circ}$ to zero.

So, while Luminet et al. (2003)'s statistical estimates of $C_{1}$ values do suggest that $\Omega_{\mathrm{tot}} \approx 1.013$ and $\alpha \approx 35^{\circ}$, it is clear that smaller $\Omega_{\text {tot }}$ values and circle sizes should be investigated.

In Figs. 2 and 3, the dependence of $\Omega_{\text {tot }}$ and $\Omega_{\mathrm{m}}$ on $\alpha$ in the angular range found to be of interest in the WMAP data is illustrated. Given the fact that the Universe is anisotropic (we clearly do not live in a true FLRW universe; we live in 


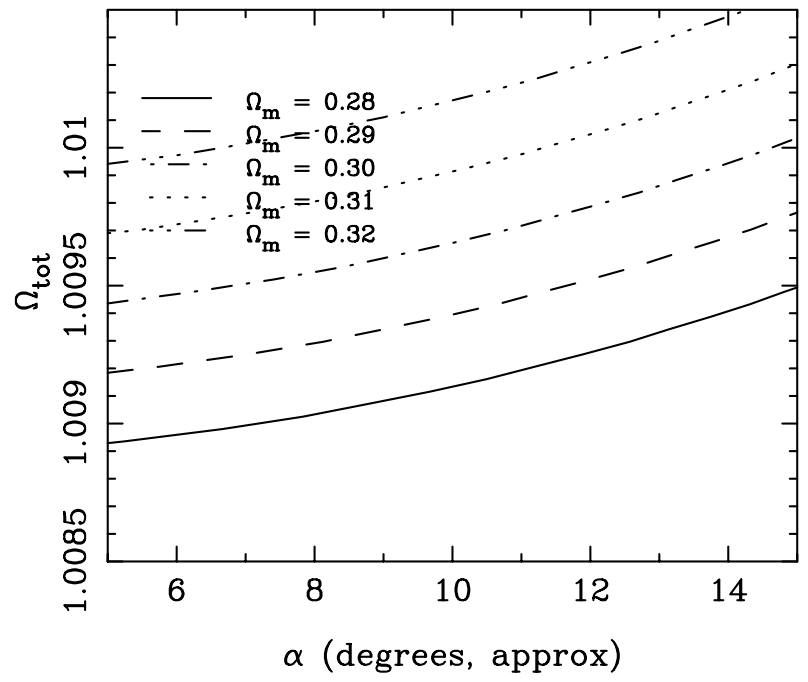

Fig. 2. An inversion of Fig. 1, focussing on the angles found to be of interest in the WMAP ILC map. Independently of $\Omega_{\mathrm{m}}$, the slope in the range $5^{\circ}<\alpha<15^{\circ}$ is $\mathrm{d} \Omega_{\text {tot }} / \mathrm{d} \alpha \approx 6 \times 10^{-5} \mathrm{deg}^{-1}$.

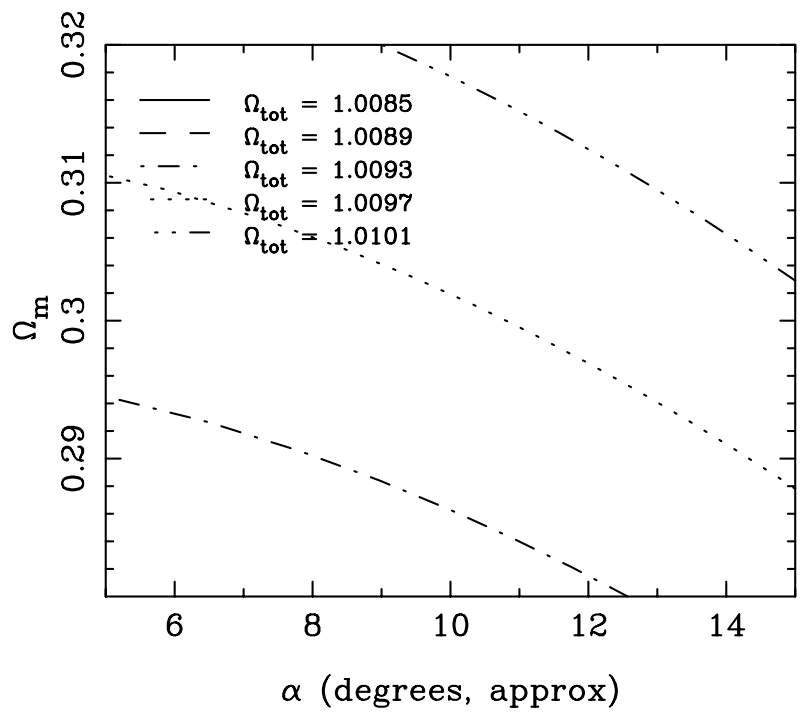

Fig. 3. Dependence of $\Omega_{\mathrm{m}}$ on $\alpha$, similarly to Fig. 2. The slope in the range $5^{\circ}<\alpha<15^{\circ}$ is $\mathrm{d} \Omega_{\mathrm{m}} / \mathrm{d} \alpha \approx 2.2 \times 10^{-3} \mathrm{deg}^{-1}$, with only weak dependence on $\Omega_{\text {tot }}$ over this interval.

a perturbed FLRW universe) some variation in the size of circles could be expected due to perturbations in the values of $\Omega_{\mathrm{m}}$ and $\Omega_{\text {tot }}$.

The variation shown in Fig. 3,

$\mathrm{d} \Omega_{\mathrm{m}} / \mathrm{d} \alpha \approx 2.2 \times 10^{-3} \mathrm{deg}^{-1}$,

is clearly much larger than temperature fluctuations on scales of about a radian $(l \sim 2-3)$.

However, the variation in $\Omega_{\text {tot }}$,

$\mathrm{d} \Omega_{\text {tot }} / \mathrm{d} \alpha \approx 6 \times 10^{-5} \mathrm{deg}^{-1}$,

is only slightly larger than that indicated by temperature fluctuations. Moreover, the unknown physics of the cosmological constant (or quintessence) makes it hard to a priori exclude any fluctuations in $\Omega_{\Lambda}$, especially with an amplitude so close to that of matter fluctuations.

Hence, a variation in circle size of about one degree would be consistent with fluctuations in $\Omega_{\Lambda}$ on scales of about a radian of amplitude about $6 \times 10^{-5}$.

Along with restrictions in practical computing power, this motivates a search strategy and smoothing on a scale of about one degree.

\subsection{Parameter space}

The space of possible orientations of a dodecahedron (centred on the observer) and possible circle matches is defined by five parameters: three for the orientation of the dodecahedron, one for the mapping between faces, and one for the circle radius.

The orientation of the dodecahedron depends on three continuous free parameters. The definition used here includes the galactic longitude and latitude $\left(l^{\mathrm{II}}, b^{\mathrm{II}}\right)$ of one face centre, and a third parameter allowing a rotation of up to $\theta=2 \pi / 5=72^{\circ}$ around the axis defined by the first centre and its opposite. The zero point of the rotation $\theta$ is arbitrary.

A given triple $\left(l^{\mathrm{II}}, b^{\mathrm{II}}, \theta\right)$ can equivalently be written as a quadruple $\left(l_{1}^{\mathrm{II}}, b_{1}^{\mathrm{II}}, l_{2}^{\mathrm{II}}, b_{2}^{\mathrm{II}}\right)$ representing two non-opposite face centres, which uniquely define the same orientation.

The mapping of one copy of the fundamental domain to the next, or what is most interesting in the present case, the matching of one face of the dodecahedron to its matching face, has one free binary parameter, $\gamma$, the handed-ness of the screw motion of the mapping (which is a Clifford translation). For a given triple $\left(l^{\mathrm{II}}, b^{\mathrm{II}}, \theta\right)$, the screw motion can either be lefthanded or right-handed. The amplitude of the screw motion is $\pi / 5=36^{\circ}$, equal to the in-diameter of the fundamental domain in units of the curvature radius.

For a rotation of $\gamma \pi / 5$, the only physically valid values are $\gamma= \pm 1$.

The remaining parameter is that discussed above, the angular radius of a circle, $\alpha$.

Putting these together, these can be written as fivedimensional parameter space:

$\left(l^{\mathrm{II}}, b^{\mathrm{II}}, \theta, \gamma, \alpha\right)$,

where four parameters are continuous variables and one of the parameters, $\gamma$, has only two physically possible values, \pm 1 .

The binary parameter provides an easy method for providing a control test which should demonstrate the likely distribution of values of statistical measures of circle identity for false matches.

By setting the Clifford translation as a translation plus a zero rotation, i.e. setting $\gamma=0$, rather than a translation plus a $\pm 36^{\circ}$ rotation, an extra 4-plane in parameter space, in which there is sure to be no genuine signal, is provided.

So, this extra 4-plane is tested here.

Strictly speaking, values of $\left(l^{\mathrm{II}}, b^{\mathrm{II}}\right)$ only need to be searched over one-twelth of the sphere, since outside of one spherical pentagon of the spherical dodecahedron, redundant points would be searched. For simplicity, the search is made for $b^{\mathrm{II}}>+50^{\circ}$, so that some redundant points are expected. 
The full parameter search space is:

$0^{\circ} \leq l^{\mathrm{II}} \leq 360^{\circ}$

$50^{\circ} \leq b^{\mathrm{II}} \leq 90^{\circ}$

$0^{\circ} \leq \theta \leq 72^{\circ}$

$\gamma \in\{0,-1,+1\}$

$1^{\circ} \leq \alpha \leq 40^{\circ}$,

where the zero screw motion is a control experiment which can only give false detections.

\subsection{Correlation and difference statistics}

For a given triple $\left(l^{\mathrm{II}}, b^{\mathrm{II}}, \theta\right), \alpha$, and the handed-ness of the screw motion, there are six pairs of circles which should have identical temperature fluctuations if there were only the naïve SachsWolfe effect, no foregrounds and no other statistical or systematic sources of noise.

Here, the statistic of Cornish et al. (1998) is used as the correlator,

$S \equiv \frac{\left\langle 2\left(\frac{\delta T}{T}\right)_{i}\left(\frac{\delta T}{T}\right)_{j}\right\rangle}{\left\langle\left(\frac{\delta T}{T}\right)_{i}^{2}+\left(\frac{\delta T}{T}\right)_{j}^{2}\right\rangle}$,

where $i$ and $j$ are the hypothetically multiply imaged locations in space-time. The mean is calculated using linearly interpolated temperature fluctuations in the ILC at intervals of 0.5 great circle degrees.

This is essentially a two-point autocorrelation function normalised by the variance.

The other statistic used is an rms difference statistic:

$\sigma^{2} \equiv\left\langle\left[\left(\frac{\delta T}{T}\right)_{i}-\left(\frac{\delta T}{T}\right)_{j}\right]^{2}\right\rangle$,

using the same notation.

Both statistics are means for all six circle pairs for the given point in parameter space $\left(l^{\mathrm{II}}, b^{\mathrm{II}}, \theta, \pm 1, \alpha\right)$.

For genuine matched circles, $S$ should be larger than for surrounding regions in parameter space, while $\sigma$ should be smaller than for surrounding regions.

\section{Results}

\subsection{Search}

The full parameter space (Eq. (8)) is searched with a resolution of $2^{\circ}$. Since the parameter space is large, not all values of $S$ and $\sigma$ are stored. Instead, over the $\theta$ dimension, values of $S$ and $\sigma$ are stored for the two values of $\theta$ which maximise $S$ and minimise $\sigma$ respectively. These two values of $\theta$ may be, but are not necessarily, identical. Cornish et al. (2004) write $S_{\max }$ to indicate that $S$ is a maximum value, not a mean value, but this is not strictly identical to the value used here, since values of both $S$ and $\sigma$ are stored for both the cases of maximum $S$ and minimum $\sigma$.

Taking the same approach as Cornish et al. (2004), the values of $S$ and $\sigma$ are plotted as a function of $\alpha$, for the Clifford translations of both orientations, i.e. rotations of $\pm 36^{\circ}$ and for a zero rotation.

These values are shown in Figs. 4-9.

There is clearly a cluster of points at $\sim 10^{\circ}$ which show excessively high values of $S$ (Fig. 4) and excessively low values of $\sigma$ (Fig. 7) for the left-handed rotation, in contrast to the general distributions of these statistics in the right-handed and zero (unphysical) cases.

The general increase in the value of $S$ as $\alpha$ decreases is similar to its behaviour in the figures of Cornish et al. (2004), and consistently, there is a corresponding decrease in $\sigma$ values as $\alpha$ decreases.

Could this cluster of points indicate a real signal?

If they correspond to a real signal, spread out over about a degree due to smoothing of the ILC map, to origin of the signal from the naïve Sachs-Wolfe effect at super-degree scales, and possibly due to intrisic variations in $\Omega_{\Lambda}$ of $\Delta \Omega_{\Lambda} \sim 6 \times 10^{-5}$ (Eq. (6)), then

(i) they should represent adjacent points in parameter space $\left(l^{\mathrm{II}}, b^{\mathrm{II}}, \theta, \pm 1, \alpha\right)$, and

(ii) each of the six individual circle pairs should show excess correlations and low differences near the optimal point.

\section{2. (i) location of the $\sim 10^{\circ}$ excess}

The twenty points with the highest values of $S$ in the range $5^{\circ}<\alpha<15^{\circ}$ all lie in one of the two ranges

$l^{\mathrm{II}}=253 \pm 4^{\circ}$

$b^{\mathrm{II}}=64 \pm 1^{\circ}$

$\theta=57.0^{\circ}$

(the point with the lowest $S$ value is at $59.0^{\circ}$ )

or

$l^{\mathrm{II}}=50 \pm 3.5^{\circ}$

$b^{\mathrm{II}}=51.0^{\circ}$

$\theta=25 \pm 2^{\circ}$.

These two triples $\left(l^{\mathrm{II}}, b^{\mathrm{II}}, \theta\right)$ correspond to the same orientation of the dodecahedron - they are two face centres for a single orientation. As mentioned above, the full parameter space is covered a little more than once, in order to be sure that all orientations are tested.

The difference statistic $\sigma$ varies more rapidly with $\alpha$, so a narrower range around $\sim 10^{\circ}$ is required to see if $\sigma$ isolates similar points to $S$.

Of the twenty points with the lowest values of $\sigma$ in the range $8^{\circ}<\alpha<15^{\circ}$, all but four lie in the ranges of Eqs. (11) and (12), except for four points with

$l^{\mathrm{II}}=57.5^{\circ}$

$b^{\mathrm{II}}=80.0 \pm 1^{\circ}$

$\theta=67 \pm 2^{\circ}$

However, the four points of Eq. (13) have correlations $0.27 \leq$ $S \leq 0.30$, much lower than for the other sixteen points, which 


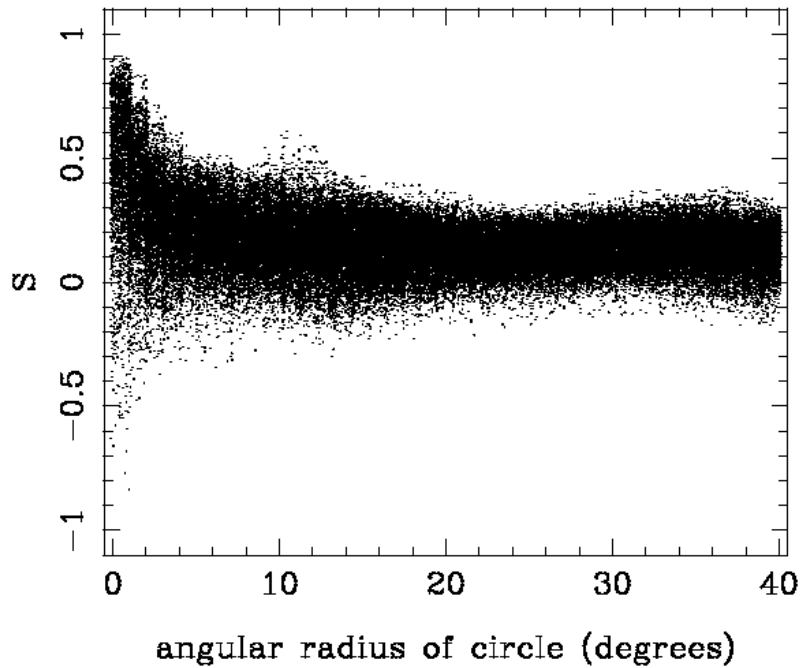

Fig. 4. Values of the correlation statistic $S$ (Eq. (9)) as a function of matched circle angular radius $\alpha$ for each pair of six matched circles implied by an orientation of the fundamental dodecahedron, where the rotation of the Clifford translation (screw motion) mapping from a circle to its image is $-36^{\circ}$ (left-handed). The values of $\alpha$ are discrete, but a small random offset in $\alpha$ filling the $0.5^{\circ}$ interval between discrete values is used for to better display individual points. The intervals in $l^{\mathrm{II}}, b^{\mathrm{II}}$ and $\theta$ are $2^{\circ}$. Points with excess correlation appear at $\sim 10^{\circ}$.

have $0.50 \leq S \leq 0.61$. This solution is not a face centre of the same dodecahedron as Eqs. (11) and (12).

Clearly, only the solution (11) and (12) is simultaneously indicated by both statistics.

\section{3. (ii) individual circle pairs}

If the $\sim 10^{\circ}$ signal is really due to the Poincare dodecahedral topology, then it should appear independently in each of the six circle pairs. If it is due to non-Gaussian and/or non-Poissonian properties of the ILC, it is unlikely to appear in all six pairs.

To test this, the correlation statistic $S$ is calculated separately for each of the six individual pairs within the range

$l^{\mathrm{II}}=253 \pm 5^{\circ}$

$b^{\mathrm{II}}=65 \pm 1^{\circ}$

$\theta=57.0 \pm 1^{\circ}$,

with a $0.5^{\circ}$ resolution.

Since the domain in most parameters is small, the mode for any given $\alpha$ value is plotted rather than a scatter plot of the individual values.

Figures 10-12 clearly show that the peak correlation at around $10^{\circ}$ is present in all six circle pairs for the left-handed screw motion.

Correlations for a few individual peaks reach similar values for the right-handed and zero (unphysical) screw motions, but not for all six pairs simultaneously.

The maximum of the individual curves in Fig. 10 is used to estimate the circle radius.

As shown in Table 1, these radii all agree to within one degree, i.e. $\alpha=11 \pm 1^{\circ}$.

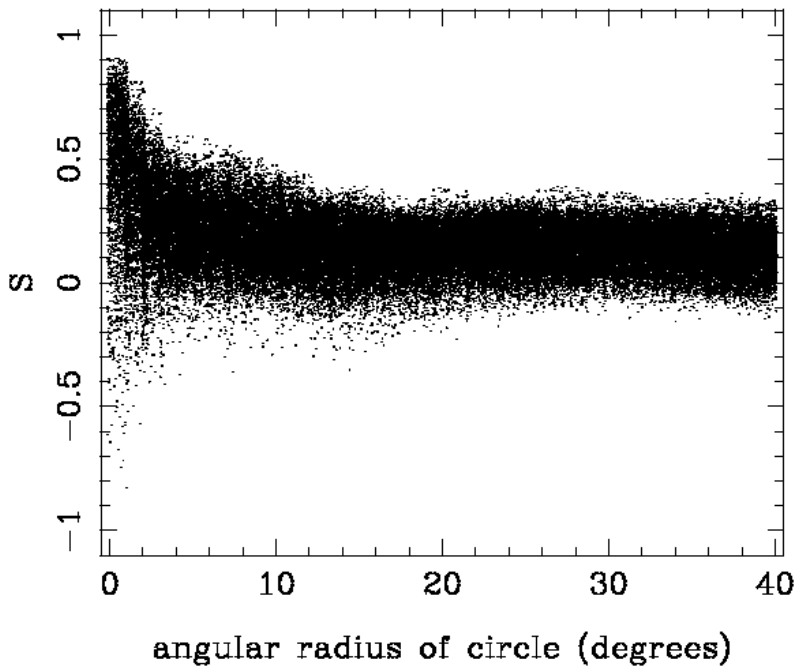

Fig. 5. As for Fig. 4, for a right-handed rotation. If a signal is present, it should be present for a left-handed rotation or a right-handed rotation, but not for both.

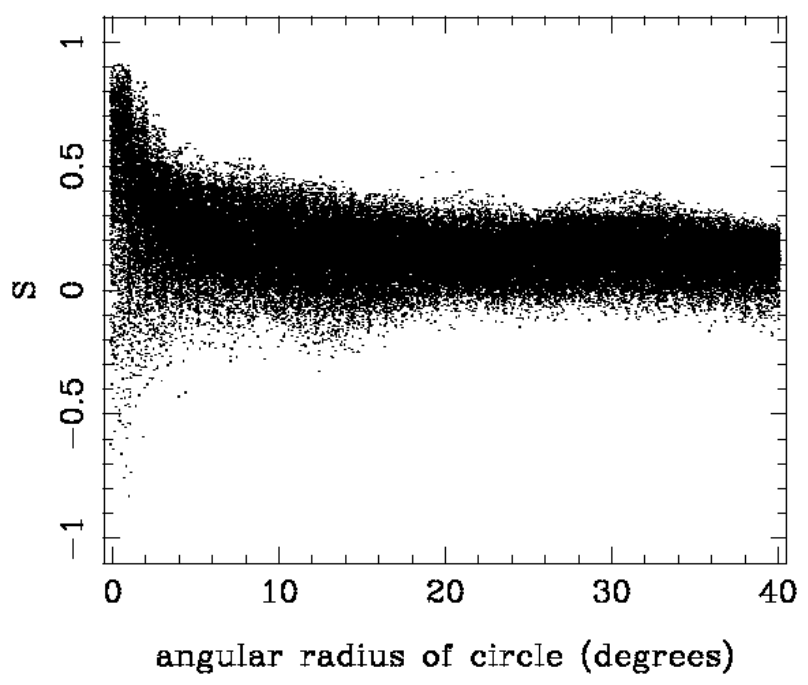

Fig. 6. As for Fig. 4, for a zero rotation. This is unphysical, so only noise signals should be present.

\subsection{Matched circles}

What are the actual values of the temperature fluctuations along the identified circles? The identified circles for the central values listed in Eq. (16) are shown in Figs. 13-18.

These clearly show that temperature values around these circles are highly correlated.

\subsection{Point source catalogue}

If the matched circles are due to topology, then any foreground point sources close to them in celestial position should be present in one circle but not the other.

Of the 208 point sources listed in the WMAP point source catalogue (Bennett et al. 2003), only one is within $0.5^{\circ}$ of the matched circle solution. This source is GB6 J1635+3808 at $\left(l^{\mathrm{II}}=61.1^{\circ}, b^{\mathrm{II}}=42.3^{\circ}\right)$ and is located within $0.1^{\circ}$ of the circle shown by the solid curve in Fig. 14. 


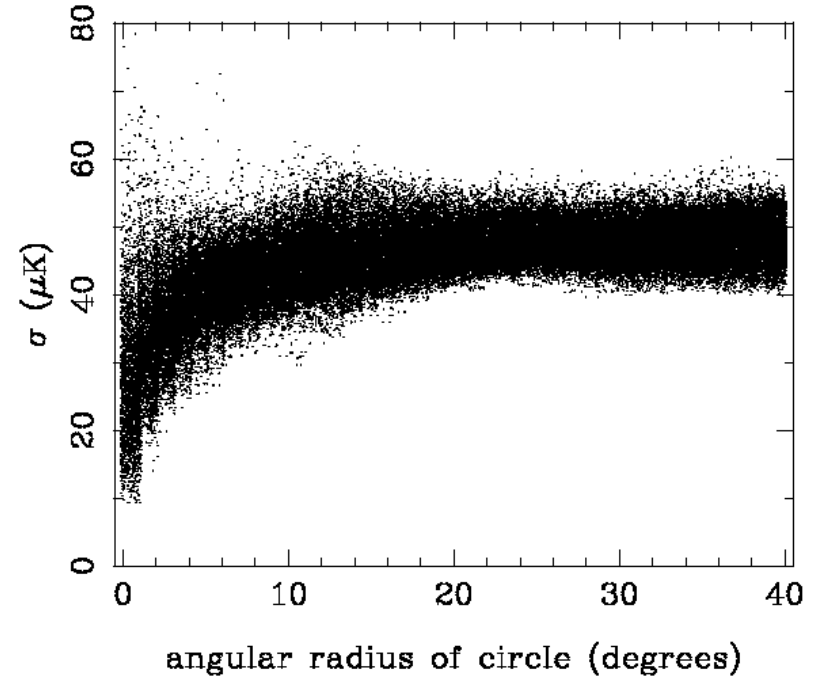

Fig. 7. As for Fig. 4, showing values of the difference statistic $\sigma$ (Eq. (10)) as a function of matched circle angular radius $\alpha$ for each pair of six matched circles implied by an orientation of the fundamental dodecahedron, again for a left-handed rotation. A cluster of points with smaller differences $\sigma$ than the general distribution is visible at $\sim 10^{\circ}$.

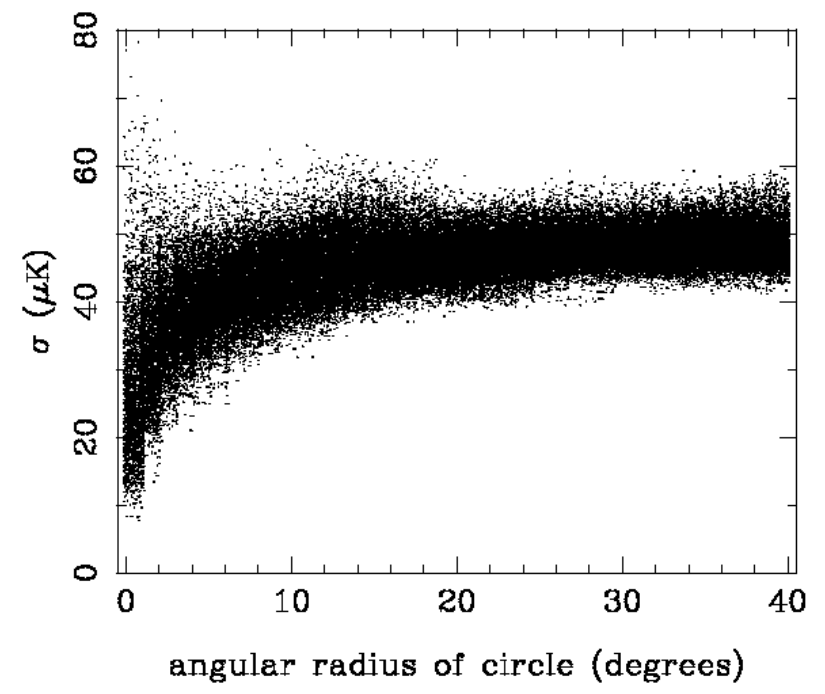

Fig. 8. As for Fig. 7, for a right-handed rotation.

Table 1. Sky positions of the six face centres for the dodecahedron which shows excess values of the correlation statistic $S$. A face number $i$, galactic longitude, latitude and estimated circle radius $\alpha$ (all in degrees) are listed. The other 6 faces are directly opposite. The orientation of the $36^{\circ}$ screw motion between faces is left-handed.

\begin{tabular}{cccc}
\hline \hline$i$ & $l^{\mathrm{II}}$ in $^{\circ}$ & $b^{\mathrm{II}}$ in $^{\circ}$ & $\alpha$ in $^{\circ}$ \\
\hline 1 & 252.4 & 64.7 & 9.8 \\
2 & 50.6 & 50.8 & 10.7 \\
3 & 143.8 & 37.8 & 10.7 \\
4 & 207.5 & 9.5 & 10.7 \\
5 & 271.0 & 2.7 & 11.8 \\
6 & 332.8 & 25.0 & 10.7 \\
\hline
\end{tabular}

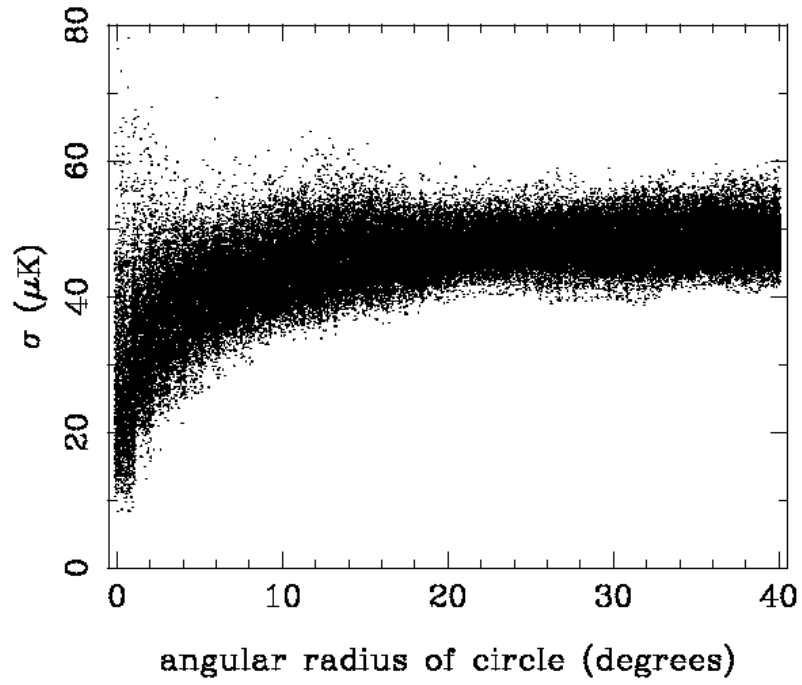

Fig. 9. As for Fig. 7, for a zero rotation.

The point source appears to be present in the curve (at position along the circle of about $1000 \mathrm{~h}^{-1} \mathrm{Mpc}$, shown by a pair of overlapping circles). A removal of flux in order to correct for the presence of this point source would improve the match between the circles.

\section{Discussion and conclusions}

It is clearly premature to claim a highly significant detection of the topology of the Universe based on just one simple analysis of the first year WMAP ILC cosmic microwave background map.

However, the plots are striking and it seems prudent to release them to the scientific community while a companion paper is prepared with formal statistical analyses.

Whether or not the matched circles found are just coincidence or due to global geometry, it is clear that temperature fluctuations around 12 dodecahedrally spaced circles of radius $11 \pm 1^{\circ}$ in the WMAP ILC map correlate unusually well in their respective pairs when a phase shift of $36^{\circ}$, corresponding to a left-handed screw motion, is applied.

If simulations can show that this is a fairly likely occurrence due to Gaussian fluctuations in an infinite flat universe, then this would show that genuine matched circles will be even harder to distinguish from spurious detections than was previously thought.

However, Vielva et al. (2004) have found non-Gaussian fluctuations at about $10^{\circ}$ - extremely close to radius of the matched circle radius. While it is not obvious how this nonGaussianity detection should relate to the radius of matched circles, since correlations are between circles, not along individual circles, this would also complicate simulations, since they would need to be consistent with observational analyses like this one.

Cornish et al. (2004) avoided circles of radii smaller than $25^{\circ}$ because of the risk of false positives, and carried out extensive simulations of what signal would be expected from a genuinely multiply connected universe. 


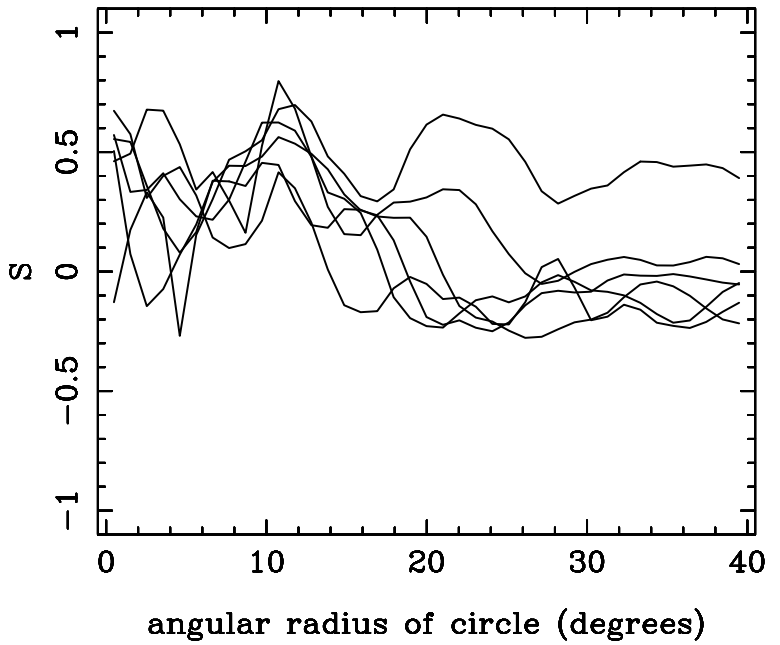

Fig. 10. Values of the correlation statistic $S$ calculated for the six individual pairs of circles for dodecahedrons with orientations in the apparently favoured range (Eq. (16)), shown as the mode at each circle radius $\alpha$. This is for a left-handed screw motion. All six pairs have high correlations at around $\sim 10^{\circ}$.

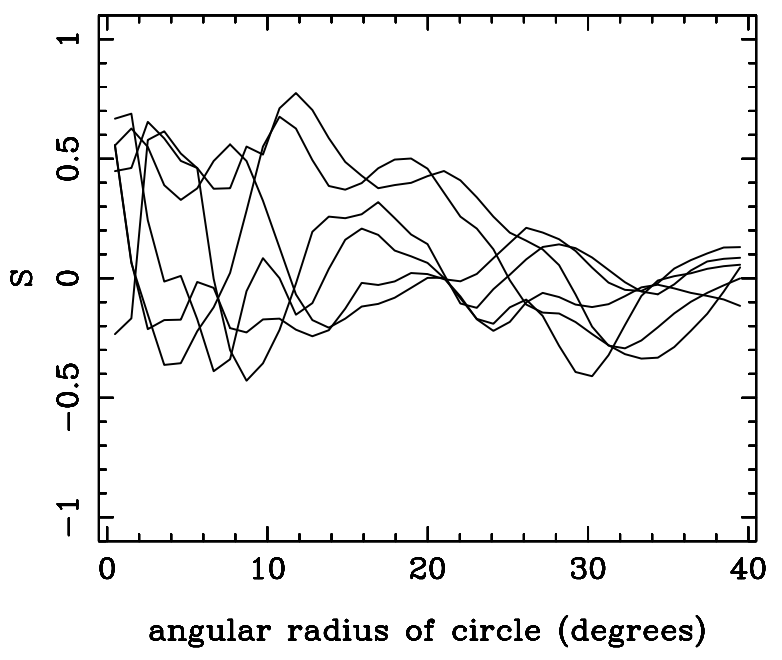

Fig. 11. As for Fig. 10, for a right-handed screw motion. Although correlations of some circle pairs are high, there is no case where all six are high.

However, these simulations risk the problem of cosmic variance and logical circularity. If the Universe really is detectably multiply connected, then it would be fairly reasonable that the density perturbations (eigenmodes) on the largest scales are somewhat affected by the global physics of the Universe, so that modelling them on the basis of Gaussianity and random phases may lead to statistical statements which are incorrect, because they talk about an ensemble of likely universes with different statistical properties to the real Universe.

The cosmic variance problem is that our actual Universe is just one realisation - the number of perturbations on large scales are too small for the large number theorem to make statistics of ensembles valid.

An apparently strong heuristic argument against these matches being physical is the need for extreme fine-tuning. If the claimed matched circles are due to topology, then the

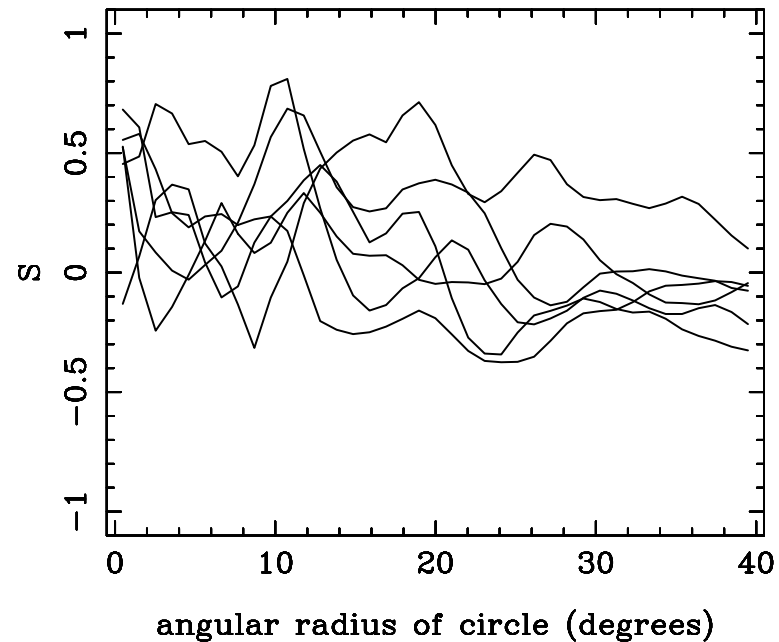

Fig. 12. As for Fig. 10, for a zero (unphysical) screw motion. Although correlations of some circle pairs are high, there is no case where all six are high.

in-radius of the Universe happens by chance to be about $\cos \left(11^{\circ}\right) \approx 98 \%$ of the distance to the surface of last scattering. Intuitively, this is difficult to accept.

However, as pointed out by Luminet et al. (2003), in the case of a positively curved Universe, especially if $\Omega_{\text {tot }} \approx 1.01$, there is necessarily a fine-tuning, since the curvature scale is only about three times the matter-dominated horizon size.

Moreover, the non-zero cosmological constant, $\Omega_{\Lambda} \approx 0.7$, is well established observationally (though see Blanchard et al. 2003; Vauclair et al. 2003, for a minority viewpoint) and definitely requires fine-tuning of some sort.

Attempts have been made to link a non-zero cosmological constant with detectable cosmic topology, but so far no obvious successes have been found.

Independently of theoretical arguments, the results presented in this paper are testable by several observationally based methods which do not require assumptions on hypothetical statistical ensembles of universes, and each should potentially be able to improve the signal if it is cosmological in origin:

(i) an attempt to separate out the naïve Sachs-Wolfe effect, the doppler component, and the integrated Sachs-Wolfe effect, and analysis at higher resolution;

(ii) improved removal of foregrounds, either independently of the hypothesis, or using the matched circles hypothesis to predict foregrounds which intervene in one circle but not the other (cf. Sect. 4.5);

(iii) polarisation data from Planck.

Similarly to the prediction by Luminet et al. (2003) that " $\Omega_{\text {tot }} \approx$ $1.013>1$ ", Fig. 1 shows that for $\Omega_{\mathrm{m}}=0.28 \pm 0.02$, the total density parameter must be $\Omega_{\text {tot }} \approx 1.010 \pm 0.001$ for these matched circles of radius $11 \pm 1^{\circ}$ to be cosmological in origin. A larger uncertainty in $\Omega_{\mathrm{m}}$ would correspondingly increase the uncertainty in $\Omega_{\text {tot }}$, but the prediction that $\Omega_{\text {tot }}>1$ remains: the fundamental dodecahedron of the Poincare dodecahedral manifold is that of a manifold of positive curvature. 


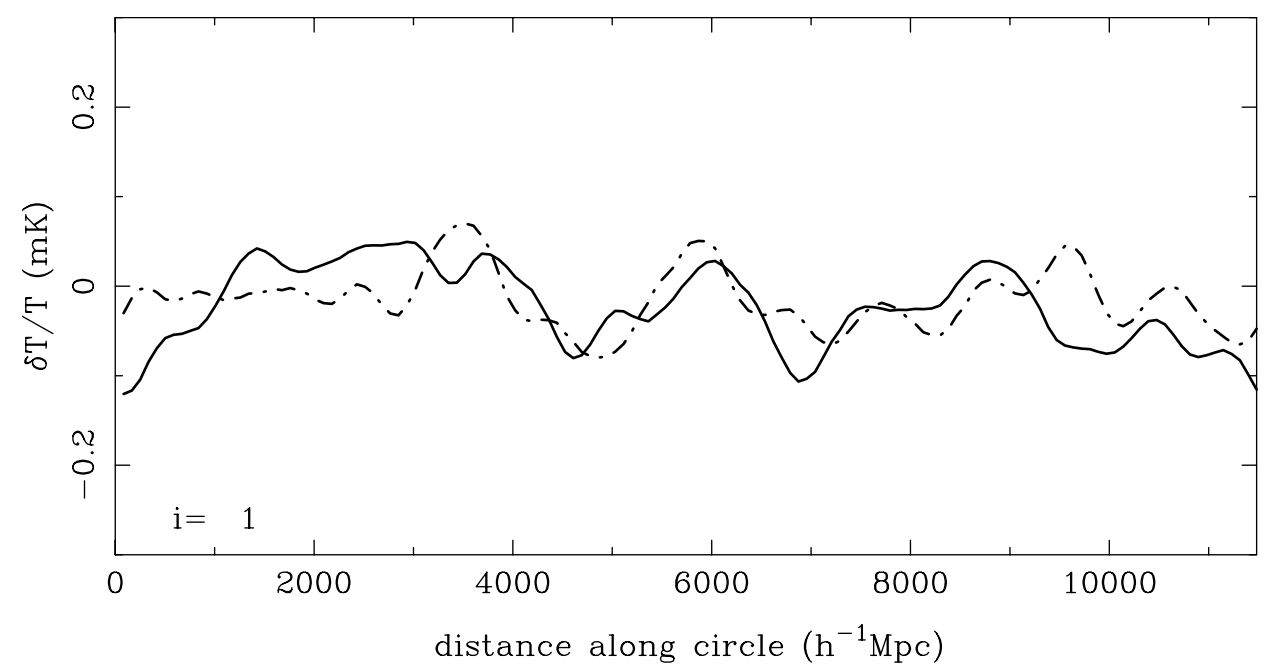

Fig. 13. Temperature fluctuations in the WMAP ILC data around an $11^{\circ}$ radius circle centred at position $i=1$ in Table 1 (continuous curve) and its opposite circle (dot-dashed circle), matched by a screw motion with a left-handed rotation of $36^{\circ}$ shown against the distance around each circle for $\left(\Omega_{\mathrm{m}}=0.3, \Omega_{\Lambda}=0.7\right)$. These distances are a slight underestimate of the true distances for the hypothesis, since Euclidean calculation is used for a "slightly" positively curved space. The two curves shown are $\delta T / T$ In the general analysis in this paper and in this and following plots, point pairs where either point lies at a galactic latitudes with $\left|b^{\mathrm{II}}\right|<2^{\circ}$ and or within $20^{\circ}$ of the galactic centre are excluded.

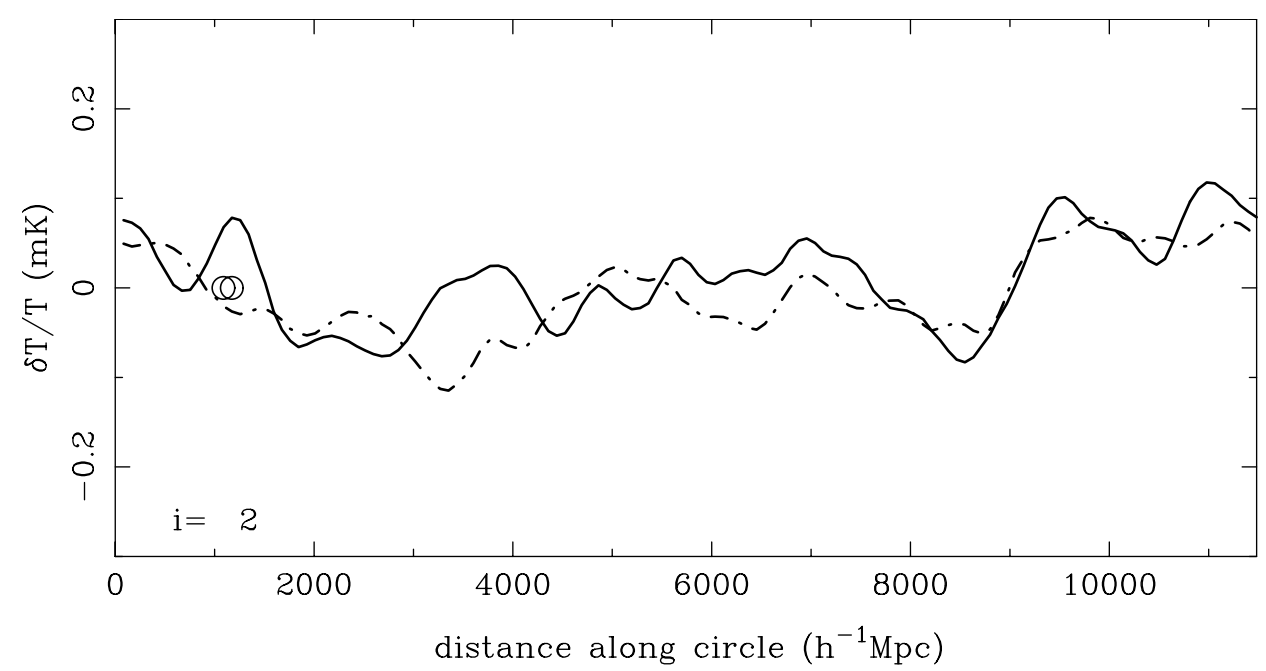

Fig. 14. As for Fig. 13, for position $i=2$ in Table 1. This is the only one of the six circle pairs for which one of the Bennett et al. (2003) point sources lies within $0.5^{\circ}$. This source is GB6 J1635+3808 at $\left(l^{\mathrm{II}}=61.1^{\circ}, b^{\mathrm{II}}=42.3^{\circ}\right)$ and is shown by a pair of overlapping circles at $($ arbitrarily) $\delta T / T=0$. Its sky position is within $0.1^{\circ}$ of the circle shown by the solid curve, suggesting that it is a contaminant present in the solid curve (Sect. 4.5).

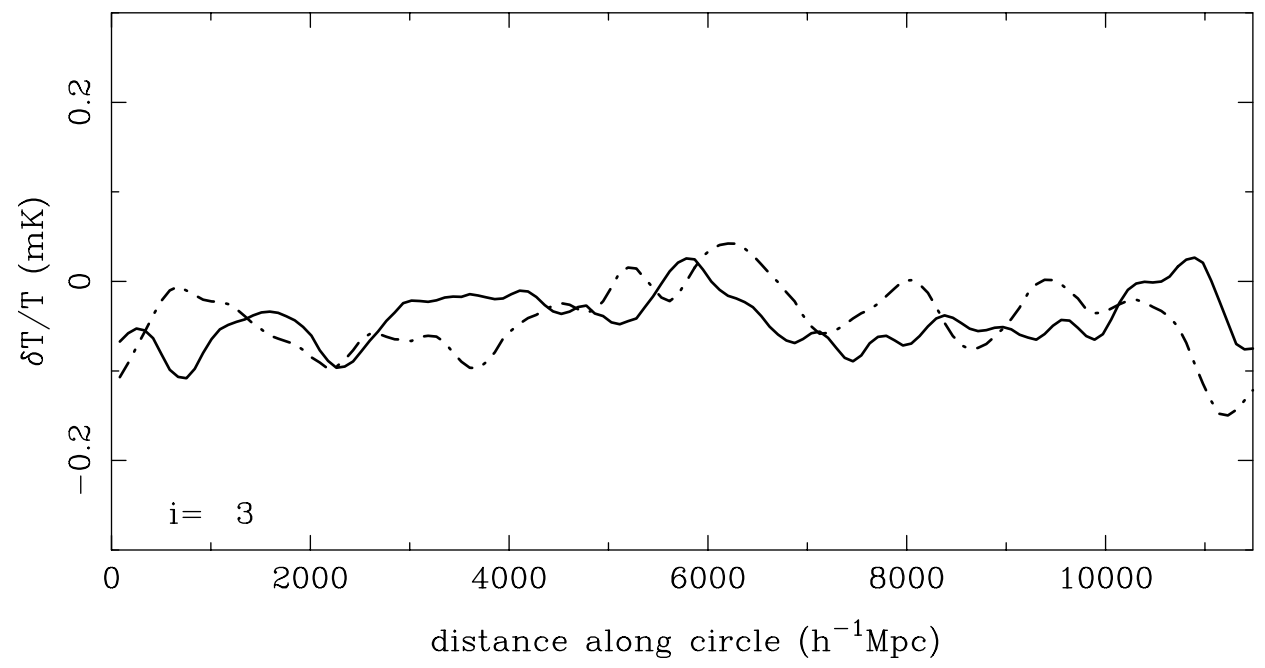

Fig. 15. As for Fig. 13, for position $i=2$ in Table 1 . 


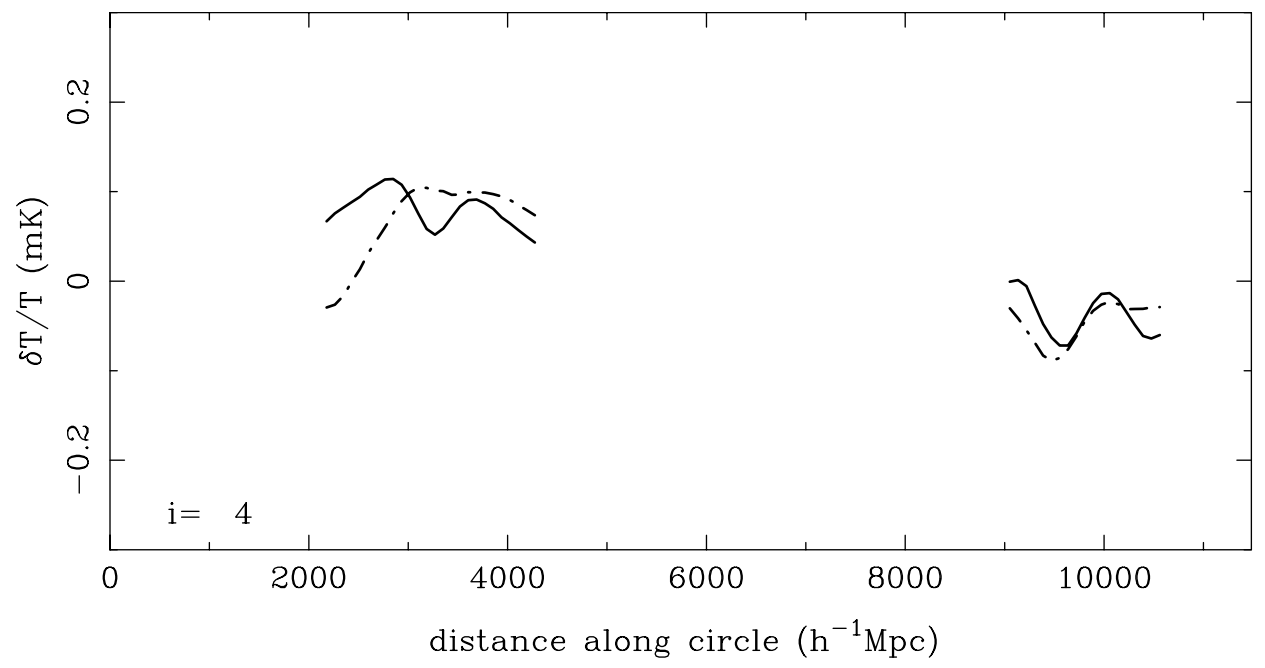

Fig. 16. As for Fig. 13, for position $i=2$ in Table 1. The exclusion due to the Galaxy mentioned in Fig. 13 excludes many points in this plot.

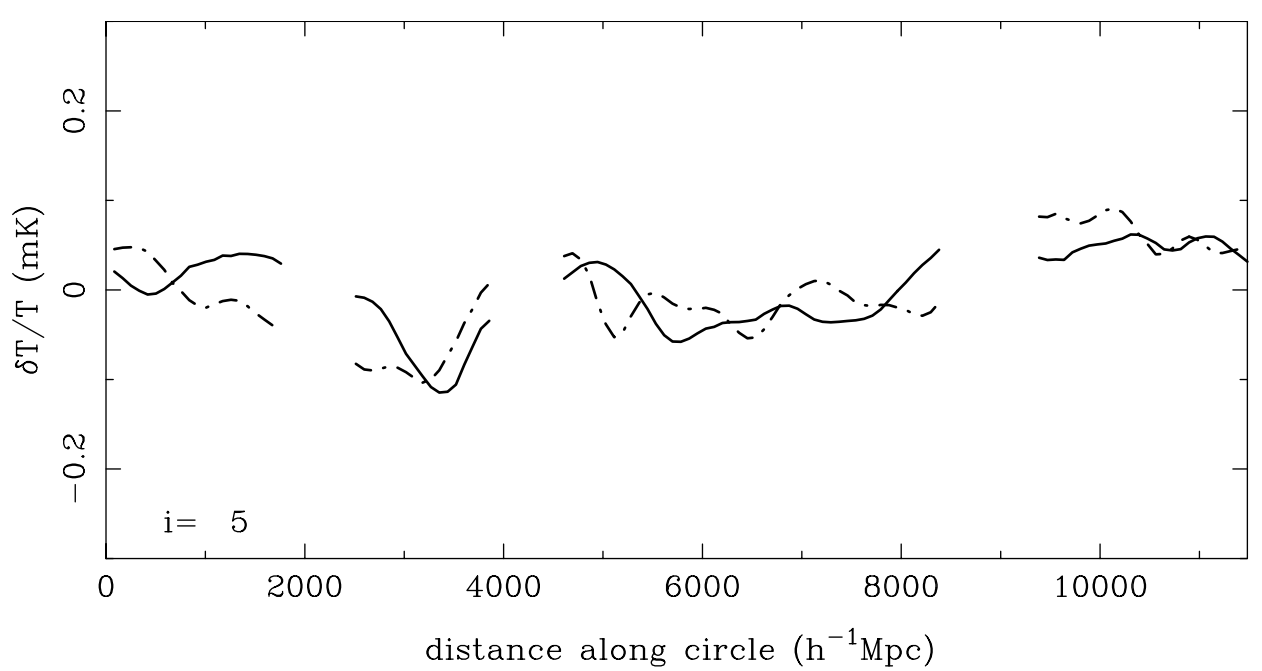

Fig. 17. As for Fig. 13, for position $i=2$ in Table 1 .

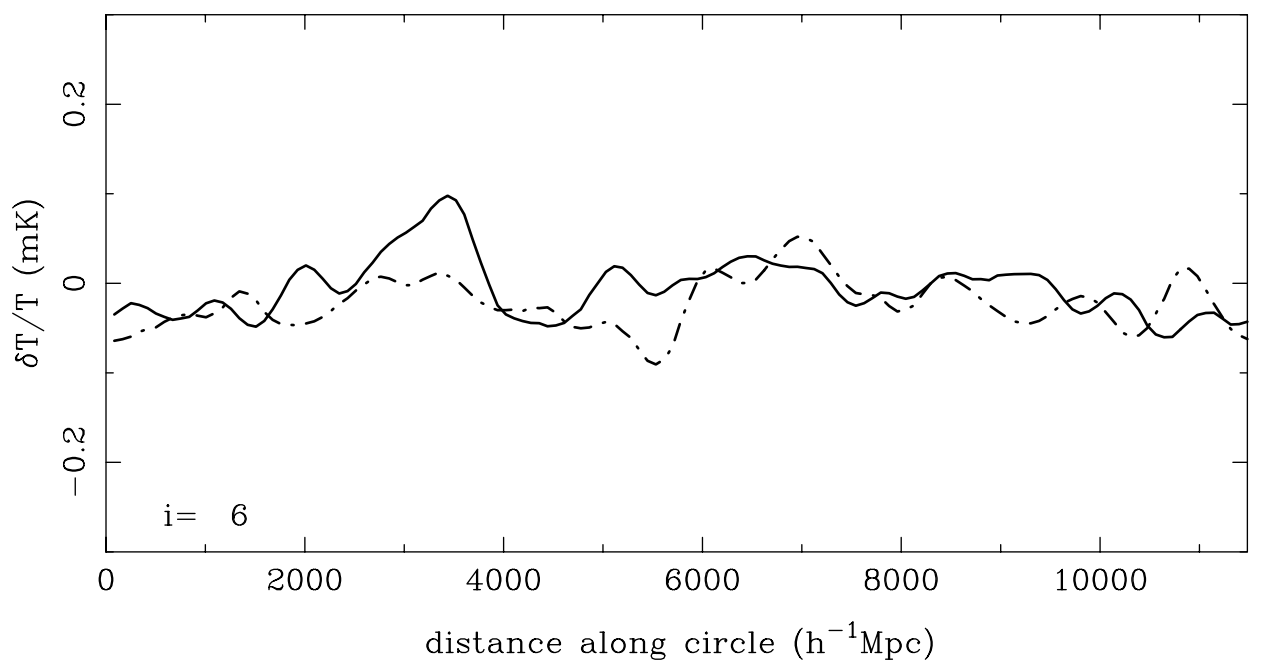

Fig. 18. As for Fig. 13, for position $i=2$ in Table 1 . 
Acknowledgements. B.F.R. is extremely grateful to Roman Juszkiewicz, Marek Demiański, Bronek Rudak and Andrzej Kus, who enabled him to continue doing cosmology research. Use was made of the WMAP data http://lambda.gsfc.nasa.gov/product/ and of the Centre de Données astronomiques de Strasbourg http://cdsads.u-strasbg.fr. Some of the results in this paper have been derived using the HEALPix (Górski et al. 1999) ${ }^{2}$. S.B. acknowledges support from KBN Grant 1P03D 01226.

\section{References}

Barrow, J. D., \& Levin, J. 2001, Phys. Rev. A, 63, 044104 Bennett, C. L., Hill, R. S., Hinshaw, G., et al. 2003, ApJS, 148, 97

Blanchard, A., Douspis, M., Rowan-Robinson, M., \& Sarkar, S. 2003, A\&A, 412, 35

Blanlœil, V., \& Roukema, B. F. 2000, Cosmological Topology in Paris 1998, Paris [arXiv:astro-ph/0010170]

Chiang, L., Naselsky, P. D., Verkhodanov, O. V., \& Way, M. J. 2003, ApJ, 590, L65

Cline, J. M., Crotty, P., \& Lesgourgues, J. 2003, JCAP, 0309, 010

Contaldi, C. R., Peloso, M., Kofman, L., \& Linde, A. 2003, J. Cosm. Astro-Part. Phys., 7, 2

Copi, C. J., Huterer, D., \& Starkman, G. D. 2003 [arXiv: astro-ph/0310511]

Cornish, N. J., Spergel, D. N., \& Starkman, G. D. 1996 [arXiv:gr-qc/9602039]

Cornish, N. J., Spergel, D. N., \& Starkman, G. D. 1998, Class. Quant. Grav., 15, 2657

Cornish, N. J., Spergel, D. N., Starkman, G. D., \& Komatsu, E. 2004, Phys. Rev. Lett., 92, 201302

de Oliveira-Costa, A., Tegmark, M., Zaldarriaga, M., \& Hamilton, A. 2004, Phys. Rev. D, 69, 063516

Efstathiou, G. 2003a, MNRAS, 343, L95

Efstathiou, G. 2003b, MNRAS, 346, L26

Efstathiou, G. 2004, MNRAS, 348, 885

Fagundes, H. V., \& Gausmann, E. 1998a

Fagundes, H. V., \& Gausmann, E. 1998b, Phys. Lett. A, 238, 235

Fagundes, H. V., \& Gausmann, E. 1999, Phys. Lett. A, 261, 235

Feng, B., \& Zhang, X. 2003, Phys. Lett. B, 570, 145

Gausmann, E., Lehoucq, R., Luminet, J.-P., Uzan, J.-P., \& Weeks, J. 2001, Classical and Quantum Gravity, 18, 5155

Giommi, P., \& Colafrancesco, S. 2004, A\&A, 414, 7

Gomero, G. I., Reboucas, M. J., \& Teixeira, A. F. F. 2000, Phys. Lett A, 275, 355

Gomero, G. I., Reboucas, M. J., \& Teixeira, A. F. F. 2001, Class. Quant. Grav., 18, 1885
Gomero, G. I., Reboucas, M. J., \& Teixeira, A. F. F. 2002, Int. J. Mod. Phys., 11, 869

Górski, K. M., Hivon, E., \& Wandelt, B. D. 1999, in Proc. of the MPA/ESO Cosmology Conf., Evolution of Large-Scale Structure, ed. A. J. Banday, R. S. Sheth, \& L. Da Costa, PrintPartners Ipskamp, NL, 37 [arXiv:astro-ph/9812350]

Lachièze-Rey, M., \& Luminet, J. 1995, Phys. Rep., 254, 135

Lehoucq, R., Lachièze-Rey, M., \& Luminet, J.-P. 1996, A\&A, 313, 339

Lehoucq, R., Luminet, J.-P., \& Uzan, J.-P. 1999, A\&A, 344, 735

Lehoucq, R., Weeks, J., Uzan, J.-P., Gausmann, E., \& Luminet, J.-P. 2002, Classical and Quantum Gravity, 19, 4683

Luminet, J., \& Roukema, B. F. 1999, in NATO ASIC Proc. 541, Theor. Obs. Cosm., 117

Luminet, J., Weeks, J. R., Riazuelo, A., Lehoucq, R., \& Uzan, J. 2003, Nature, 425, 593

Luminet, J.-P. 1998, Acta Cosmol., XXIV-1, 105

Naselsky, P. D., Doroshkevich, A. G., \& Verkhodanov, O. V. 2003, ApJ, 599, L53

Naselsky, P. D., Doroshkevich, A. G., \& Verkhodanov, O. V. 2004, MNRAS, 349, 695

Riazuelo, A., Weeks, J., Uzan, J.-P., Lehoucq, R., \& Luminet, J. 2004, Phys. Rev. D, 69, 103518

Roukema, B. F. 1996, MNRAS, 283, 1147

Roukema, B. F. 2000a, Class. Quant. Grav., 17, 3951

Roukema, B. F. 2000b, MNRAS, 312, 712

Roukema, B. F., \& Blanlœil, V. 1998, Class. Quant. Grav., 15, 2645

Roukema, B. F., \& Edge, A. C. 1997, MNRAS, 292, 105

Schwarzschild, K. 1900, Vier. d. Astr. Gess, 35, 337

Schwarzschild, K. 1998, Class. Quant. Grav., 15, 2539

Spergel, D. N., Verde, L., Peiris, H. V., et al. 2003, ApJS, 148, 175

Starkman, G. D. 1998, Class. Quant. Grav., 15, 2529

Tegmark, M., de Oliveira-Costa, A., \& Hamilton, A. 2003, Phys. Rev. D, 68, 123523

Uzan, J.-P., Lehoucq, R., \& Luminet, J.-P. 1999, A\&A, 351, 766

Uzan, J.-P., Luminet, J.-P., Lehoucq, R., \& Peter, P. 2002, Eur. J. Phys., 23, 277

Vauclair, S. C., Blanchard, A., Sadat, R., et al. 2003, A\&A, 412, L37

Vielva, P., Martinez-González, E., Barreiro, R. B., Sanz, J. L., \& Cayón, L. 2004, ApJ, 609, 22

Weeks, J. 2001, The Shape of Space, 2nd edition (Manhattan: Marcel Dekker)

Weinberg, S. 1972, Gravitation and cosmology: Principles and applications of the general theory of relativity (New York: Wiley)

\footnotetext{
${ }^{2}$ see http://www.eso.org/science/healpix/
} 